\title{
Article \\ Mechanical Loading-Driven Tumor Suppression Is Mediated by Lrp5-Dependent and Independent Mechanisms
}

\author{
Yan Feng ${ }^{1,2}$, Shengzhi Liu ${ }^{2}$, Rongrong Zha ${ }^{1,2}$, Xun Sun ${ }^{1,2}$, Kexin Li ${ }^{1,2}$, Alexander Robling ${ }^{3,4}$, Baiyan Li ${ }^{1, *}$ and \\ Hiroki Yokota $1,2,3,4,5, *$ \\ 1 Department of Pharmacology, College of Pharmacy, Harbin Medical University, Harbin 150081, China; \\ fengya@iu.edu (Y.F.); zhar@iu.edu (R.Z.); sunxun@iu.edu (X.S.); kexli@iu.edu (K.L.) \\ 2 Department of Biomedical Engineering, Indiana University Purdue University Indianapolis, \\ Indianapolis, IN 46202, USA; liu441@iu.edu \\ 3 Department of Anatomy Cell Biology and Physiology, Indiana University School of Medicine, \\ Indianapolis, IN 46202, USA; arobling@iupui.edu \\ 4 Indiana Center for Musculoskeletal Health, Indiana University School of Medicine, \\ Indianapolis, IN 46202, USA \\ 5 Simon Cancer Center, Indiana University School of Medicine, Indianapolis, IN 46202, USA \\ * Correspondence: liby@ems.hrbmu.edu.cn (B.L.); hyokota@iupui.edu (H.Y.); Tel.: +86-451-8667-1354 (B.L.); \\ +317-278-5177 (H.Y.); Fax: +86-451-8667-1354 (B.L.); +317-278-2455 (H.Y.)
}

check for

updates

Citation: Feng, Y.; Liu, S.; Zha, R.; Sun, X.; Li, K.; Robling, A.; Li, B.;

Yokota, H. Mechanical

Loading-Driven Tumor Suppression Is Mediated by Lrp5-Dependent and Independent Mechanisms. Cancers 2021, 13, 267. https://doi.org/ $10.3390 /$ cancers 13020267

Received: 12 December 2020

Accepted: 7 January 2021

Published: 13 January 2021

Publisher's Note: MDPI stays neutral with regard to jurisdictional clai$\mathrm{ms}$ in published maps and institutional affiliations.

Copyright: (C) 2021 by the authors. Licensee MDPI, Basel, Switzerland. This article is an open access article distributed under the terms and conditions of the Creative Commons Attribution (CC BY) license (https:// creativecommons.org/licenses/by/ $4.0 /)$.
Simple Summary: Advanced breast cancer and prostate cancer metastasize to varying organs including the bone. We show here that mechanical loading to the knee suppresses tumor growth in the loaded bone and the non-loaded mammary pad. Although lipoprotein receptor-related protein 5 (Lrp5) in osteocytes is necessary to induce loading-driven bone formation, loading-driven tumor suppression is regulated by Lrp5-dependent and independent mechanisms. Lrp5 overexpression in osteocytes enhances tumor suppression, but without Lrp5 in osteocytes, mechanical loading elevates dopamine, chemerin, p53, and TNF-related apoptosis-inducing ligand (TRAIL) and reduces cholesterol and nexin. Their systemic changes contribute to inhibiting tumors without Lrp5. Osteoclast development is also inhibited by the load-driven regulation of chemerin and nexin.

Abstract: Bone is mechanosensitive and lipoprotein receptor-related protein 5 (Lrp5)-mediated Wnt signaling promotes loading-driven bone formation. While mechanical loading can suppress tumor growth, the question is whether Lrp5 mediates loading-driven tumor suppression. Herein, we examined the effect of Lrp5 using osteocyte-specific Lrp5 conditional knockout mice. All mice presented noticeable loading-driven tumor suppression in the loaded tibia and non-loaded mammary pad. The degree of suppression was more significant in wild-type than knockout mice. In all male and female mice, knee loading reduced cholesterol and elevated dopamine. It reduced tumor-promoting nexin, which was elevated by cholesterol and reduced by dopamine. By contrast, it elevated p53, TNF-related apoptosis-inducing ligand (TRAIL), and chemerin, and they were regulated reversely by dopamine and cholesterol. Notably, Lrp5 overexpression in osteocytes enhanced tumor suppression, and osteoclast development was inhibited by chemerin. Collectively, this study identified Lrp5dependent and independent mechanisms for tumor suppression. Lrp5 in osteocytes contributed to the loaded bone, while the Lrp5-independent regulation of dopamine- and cholesterol-induced systemic suppression.

Keywords: breast cancer; prostate cancer; mechanical stimulation; osteocyte; Lrp5; chemerin; nexin

\section{Introduction}

Breast cancer is the second leading cause of cancer death in women worldwide. While one out of eight women suffers from breast cancer in her lifetime, treating advanced breast cancer remains a challenge [1]. Prostate cancer is the most frequent tumor found in men 
worldwide [2]. Bone metastasis is a frequent complication [3] for bone metastatic breast cancer and advanced prostate cancer [4,5]. Metastasis to bone induces pain, hypercalcemia, and the risk of bone fracture [6]. It is well known that bone is responsive to its mechanical environment and that a lack of mechanical stimulation promotes bone loss [7,8]. Loading modalities such as axial loading and whole-body vibration can increase bone mass by activating bone-forming osteoblasts $[9,10]$, as well as inhibiting bone-resorbing osteoclasts [6]. Mechanical loading also suppresses tumor-driven osteolysis by restraining bone resorption [11], inhibits the progression of myeloma in the bone [12], and induces apoptosis of breast cancer cells [13]. The molecular mechanisms of loading-driven tumor suppression and the role of Wnt signaling remains elusive.

We previously reported that osteocytic expression of low-density lipoprotein receptorrelated protein 5 (Lrp5), a co-receptor for Wnt ligands, is necessary to induce loading-driven bone formation [14-16]. It is also reported that specific gain-of-function missense mutations in Lrp5 induced protection from disuse-related bone loss by increasing osteogenic responsiveness to loading [17]. Using a mouse model of bone metastasis associated with breast cancer and prostate cancer [18], the main question we addressed in this study was whether Lrp5 in osteocytes is necessary to induce loading-driven tumor suppression. We employed mice with osteocyte-selective deletion of Lrp5 and evaluated the Lrp5-dependent and independent mechanisms involved in loading-driven tumor suppression.

Cyclic lateral loads were applied to the knees of wild-type and osteocyte-specific Lrp5 knockout mice. It is reported that knee loading not only stimulates bone formation and prevents cartilage degeneration but also promotes vessel remodeling and bone healing in the necrotic femoral head [19]. Knee loading is shown to activate endocrine and neural signaling and induce local and global responses [20,21], and we have shown that it can inhibit tumor-driven osteolysis in the mouse model of breast cancer and prostate cancer [22]. However, little is known about the role of Lrp5-mediated Wnt signaling in loading-driven tumor suppression. In this study, we examined the potential involvement of Lrp5 in osteocytes in tumor suppression at a locally loaded site of the tibia as well as at a remote non-loaded site of the mammary fat pad.

In evaluating the Lrp5-mediated and Lrp5-non-mediated mechanisms of loadingdriven tumor suppression, we examined the expression of oncogenic genes such as Runx2, Snail, and TGF $\beta$ in the context of Lrp5 overexpression and silencing. In our previous work, step aerobics was used as a means of mechanical stimulation, which significantly altered the levels of dopamine and cholesterol in human urine samples [23]. Thus, we examined their urinary and serum levels in wild-type and knockout mice before and after knee loading. We also determined the role of tumor-promoting cytokines and metalloproteinases such as nexin (Serpin peptidase inhibitor, clade E, member 2 -SERPINE2), MMP2, MMP3, and MMP9, as well as tumor-suppressing factors such as p53, TNF-related apoptosis-inducing ligand (TRAIL), and chemerin (retinoic acid receptor responder protein 2 (RARRES2)), and an apoptosis marker, cleaved caspase 3 .

Beyond evaluating suppression of tumor progression, we also measured the effects of lateral loading on the development of bone-resorptive osteoclasts by determining the expression of NFATc1, a master transcription factor for osteoclastogenesis, and cathepsin $\mathrm{K}$, a proteinase for osteoclast maturation. Tumor-induced osteolysis is amplified by the vicious cycle through tumor-osteoclast interactions [11]. The results herein supported that both Lrp5-dependent and independent mechanisms are present in loading-driven tumor suppression. Since Lrp5-mediated Wnt signaling can promote tumorigenesis and tumor progression, the tumor-suppressing role of Lrp5 in osteocytes indicates that osteocytes and tumor cells use Lrp5 signaling in distinct and opposing ways.

\section{Results}

\subsection{Inhibitory Effects of Knee Loading on Mammary Tumors}

The main aim of this study was to identify the role of Lrp5 in inducing loading-driven suppression of tumor progression. We first confirmed genotypes for the presence of Cre 
and Lrp5 floxed alleles. Mice lacking Lrp5 in osteocytes (KO mice) had Dmp1-Cre and homozygous flox alleles (Figure 1A). Using mice evenly distributed by age into four groups (Figure 1B), we evaluated the effect of daily knee loading for two weeks (1 N peak-to-peak at $2 \mathrm{~Hz}$ for $5 \mathrm{~min}$ ) on mammary tumors that were induced by the inoculation of EO771 cells into the mammary fat pad of C57BL/six female mice (Figure 1C). Knee loading reduced the tumor size and weight in the mammary fat pad in mice regardless of Lrp 5 deletion (Figure 1D; Figure S1A). The result revealed that the mammary tumor can be suppressed remotely by mechanical stimulation to the knee both in wild-type and knockout mice independent of osteocytic Lrp5.
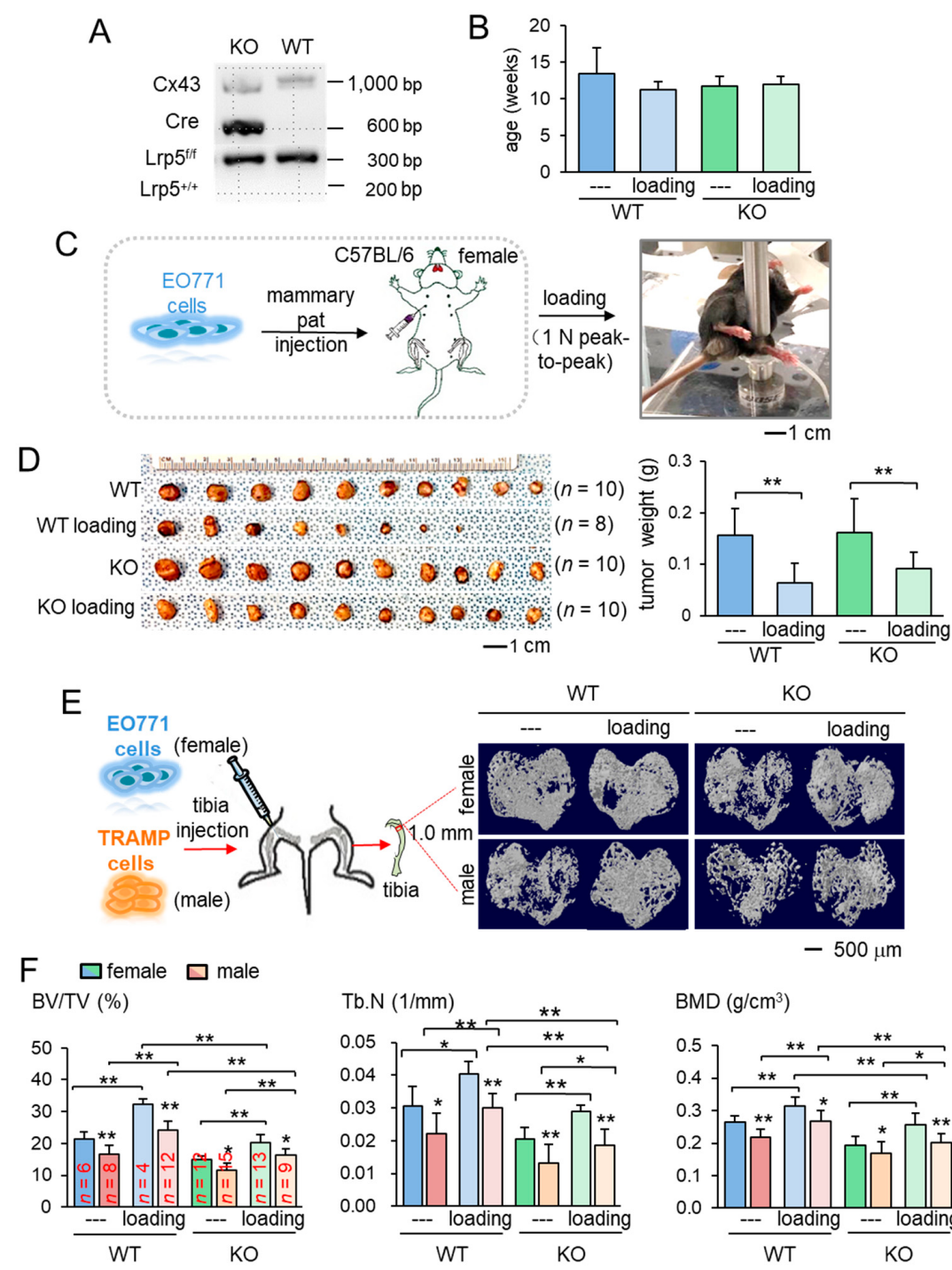

E
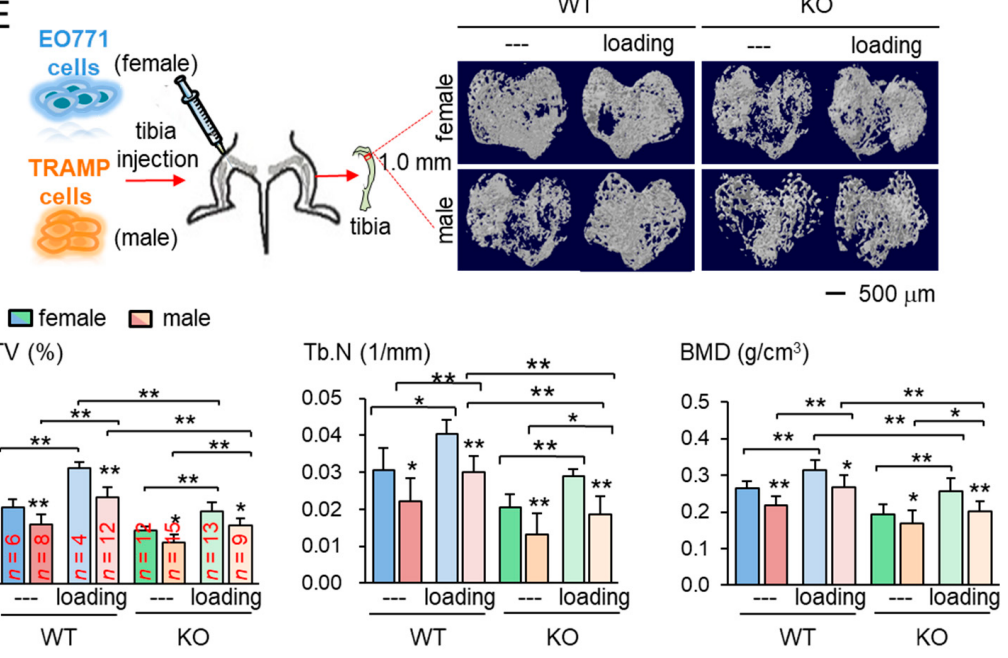

Figure 1. Loading-driven reduction in tumor progression. $\mathrm{KO}=$ knockout, and $\mathrm{WT}=$ wild-type. The single and double asterisks indicate $p<0.05$ and $p<0.01$, respectively. (A) Genotyping of wild-type and knockout mice. Cre was driven by the BMP1 promoter, and C $\times 43$ was used as an internal control. The genotypes of a pair of mice (KO and WT) were shown based on amplified PCR products, including floxed Lrp5 (f/f), and Lrp5 (+/+) alleles. (B) Age of the mice used in this study. (C) Procedure for the inoculation of EO771 mammary tumor cells and cyclic knee loading. (D) Images of mammary tumors in four groups (wild-type and knockout with and without knee loading). (E) Micro CT images of the proximal tibiae of the wild-type and knockout mice with and without knee loading. Female mice received EO771 mammary tumor cells, while male mice received transgenic adenocarcinoma of the mouse prostate (TRAMP) prostate tumor cells in their proximal tibia. (F) Three micro CT-linked parameters in the proximal tibiae in the wild-type and knockout mice. BV $/ \mathrm{TV}=$ bone volume ratio, $\mathrm{Tb} . \mathrm{N}=$ trabecular number, and $\mathrm{BMD}=$ bone mineral density separation. 


\subsection{Protection of Tumor-Invaded Tibia by Knee Loading}

We next examined the effect of knee loading on the tumor-invaded tibia. After intratibial injection of EO771 mammary tumor cells to female mice and transgenic adenocarcinoma of the mouse prostate (TRAMP) prostate tumor cells to male mice, we applied knee loading to both wild-type and knockout mice using the same loading condition in the previous experiment. We observed that daily knee loading reduced the degradation of trabecular bone in the proximal tibia (Figure 1E). Specifically, a significant increase in bone volume ratio (BV/TV), trabecular number (Tb.N), and bone mineral density (BMD) was observed by knee loading, with a reduction in the trabecular separation (Tb.Sp) (Figure 1F; Figure S1B). The result of these four parameters is consistent with the protective effect of knee loading. Also, Hematoxylin and Eosin (H\&E)-stained sagittal sections revealed that knee loading reduced the area of tumor invasion regardless of the deletion of Lrp5 in osteocytes (Figure 2A-C). Notably, although knee loading suppressed the growth of tumors in the tibia, the tumor-induced damage was more severe when Lrp5 was deleted. The loading effect on tumor areas was larger in wild-type mice than knockout mice in both females and males (Figure 2D). These results indicated that Lrp5 in osteocytes contributed to protecting bone from tumor invasion, but loading-driven suppression of tumor-induced bone loss was observed even in osteocyte-specific Lrp5 knockout mice.

The results so far showed the beneficial role of Lrp5 in osteocytes in the tumor-invaded tibia regardless of knee loading. In the absence of tumor inoculation, the benefit of knee loading to bone formation was only observed in wild-type mice. While knee loading elevated BV/TV, BMD, Tb.N with a decrease in Tb.Sp in the trabecular bone of wildtype mice, it did not induce any significant alteration in knockout mice (Figure 3A,B). Furthermore, in the cortical bone of the same proximal tibiae, knee loading increased BMD in the tibia of wild-type mice but not that of knockout mice (Figure S2). The result indicates that Lrp5 in osteocytes is necessary for inducing loading-driven bone formation, but it is not required for causing loading-driven tumor suppression.

\subsection{Loading-Dependent Alterations in Dopamine and Cholesterol in the Serum and Urine}

To address the mechanism of loading-driven tumor suppression in the absence of Lrp5 in osteocytes, serum and urine samples were collected from female mice before and $1 \mathrm{~h}$ after the application of knee loading and assayed for dopamine and cholesterol. Compared to the pre-loading samples, the post-loading samples exhibited elevated levels of dopamine and reduced the level of cholesterol in the serum and urine (Figure 3C-F). Notably, the altered levels of dopamine and cholesterol were observed irrespective of Lrp5 in osteocytes.

\subsection{Regulation of Chemerin as a Tumor Suppressor and Nexin as a Tumor Promoter}

Besides the loading-driven alterations in cholesterol and dopamine, antibody-based cytokine array analysis revealed that knee loading substantially elevated the relative expression level of chemerin and reduced the relative expression level of nexin in the serum of both wild-type and knockout mice (Figure 4A, Figure S3A). The regulation of chemerin and nexin was also observed in EO771 cells in response to dopamine and cholesterol (Figure 4B, Figure S3B). The summary of four pairs of cytokine profiles (knee loading in wild-type and knockout mice, and the responses to dopamine and cholesterol) indicated that chemerin acted as a tumor suppressor, while nexin was considered as a tumor promoter (Figure 4C). Of note, MMP2 and MMP3 also acted as tumor promoters in five out of eight cases (Figure 4C). Western blot analysis showed that the level of chemerin was elevated by dopamine and reduced by cholesterol in EO771 cells (Figure 4D). Furthermore, chemerin was upregulated and nexin was downregulated by knee loading in the serum of wild-type and knockout mice (Figure 4E). Chemerin reduced MTT-based cell viability, Transwell invasion, and scratch-based migration in EO771 cells, while nexin elevated them (Figure S4). Collectively, the result supported the possibility that knee loading suppressed tumor progression in part by systematically elevating dopamine and chemerin, while reducing cholesterol and nexin, even in the absence of Lrp5 in osteocytes. 
A

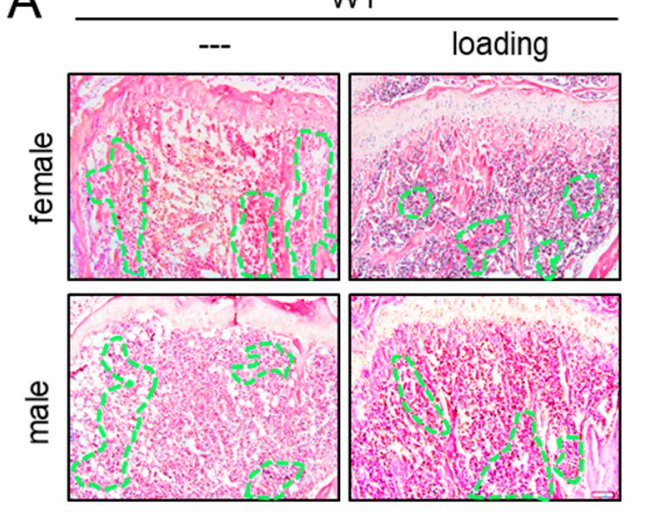

B
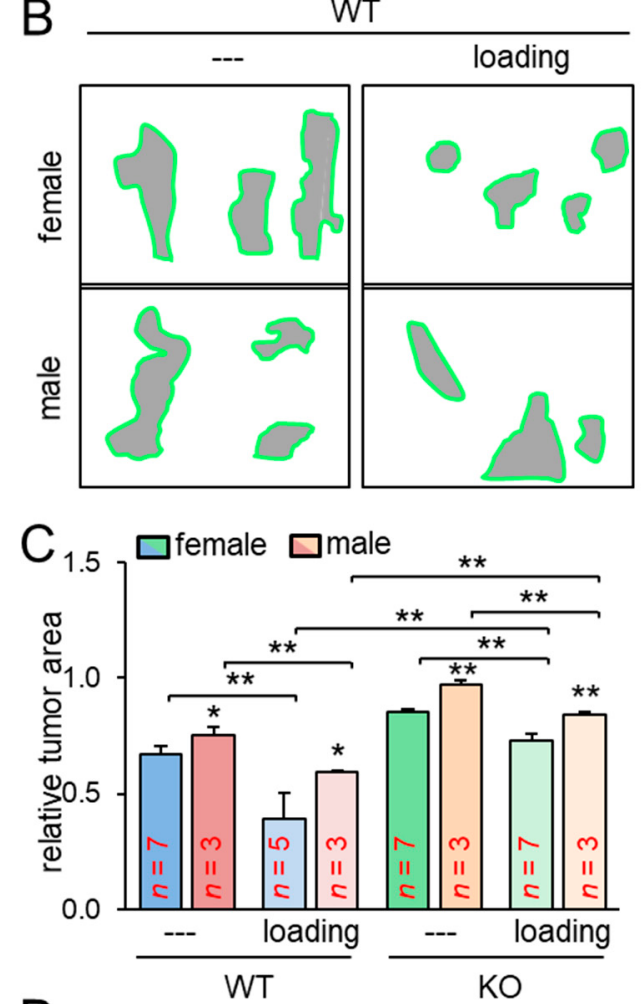
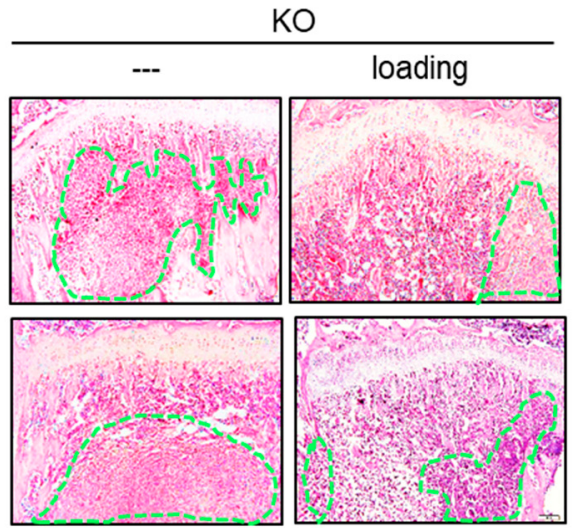

$-200 \mu \mathrm{m}$

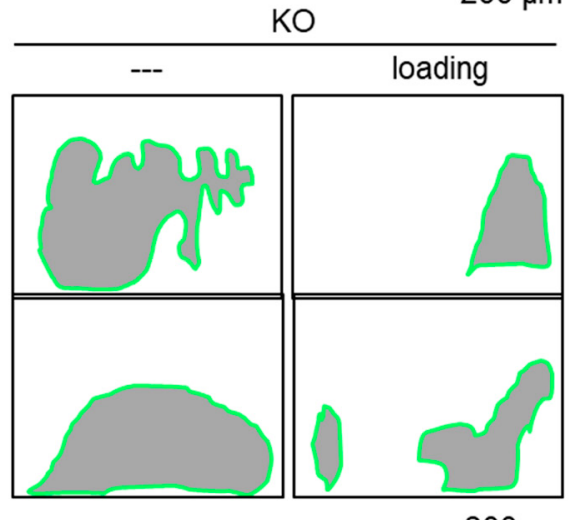

$-200 \mu \mathrm{m}$

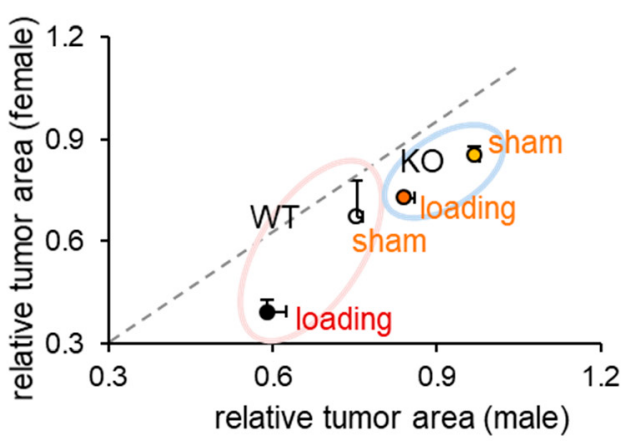

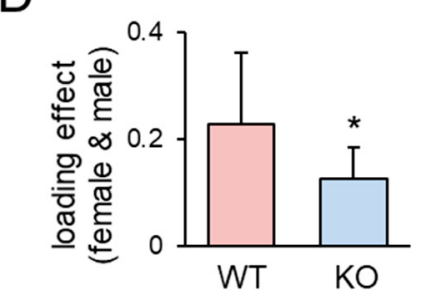
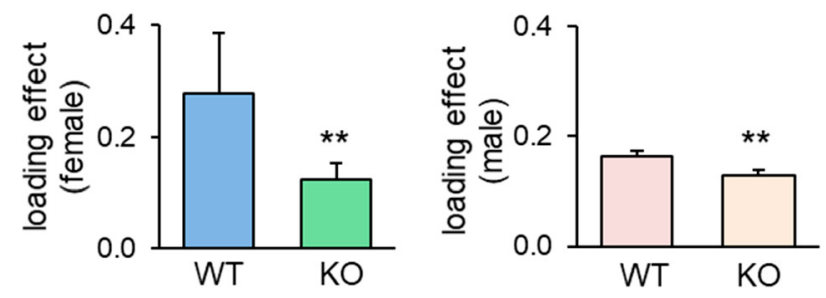

Figure 2. Reduction of the tumor-invaded area by knee loading. $\mathrm{KO}=$ knockout, and $\mathrm{WT}=$ wild-type. The single and double asterisks indicate $p<0.05$ and $p<0.01$, respectively. (A,B) H\&E-stained histological images of the proximal tibiae of the wild-type and knockout mice with and without knee loading, and the region of the tumor-invaded area. (C) Comparison of the relative tumor area in the proximal tibiae for female and male mice. (D) Comparison of the loading effects on tumor areas between wild-type and knockout mice. The first plot is for all mice, and the second and third plots are for female and male mice, respectively. 

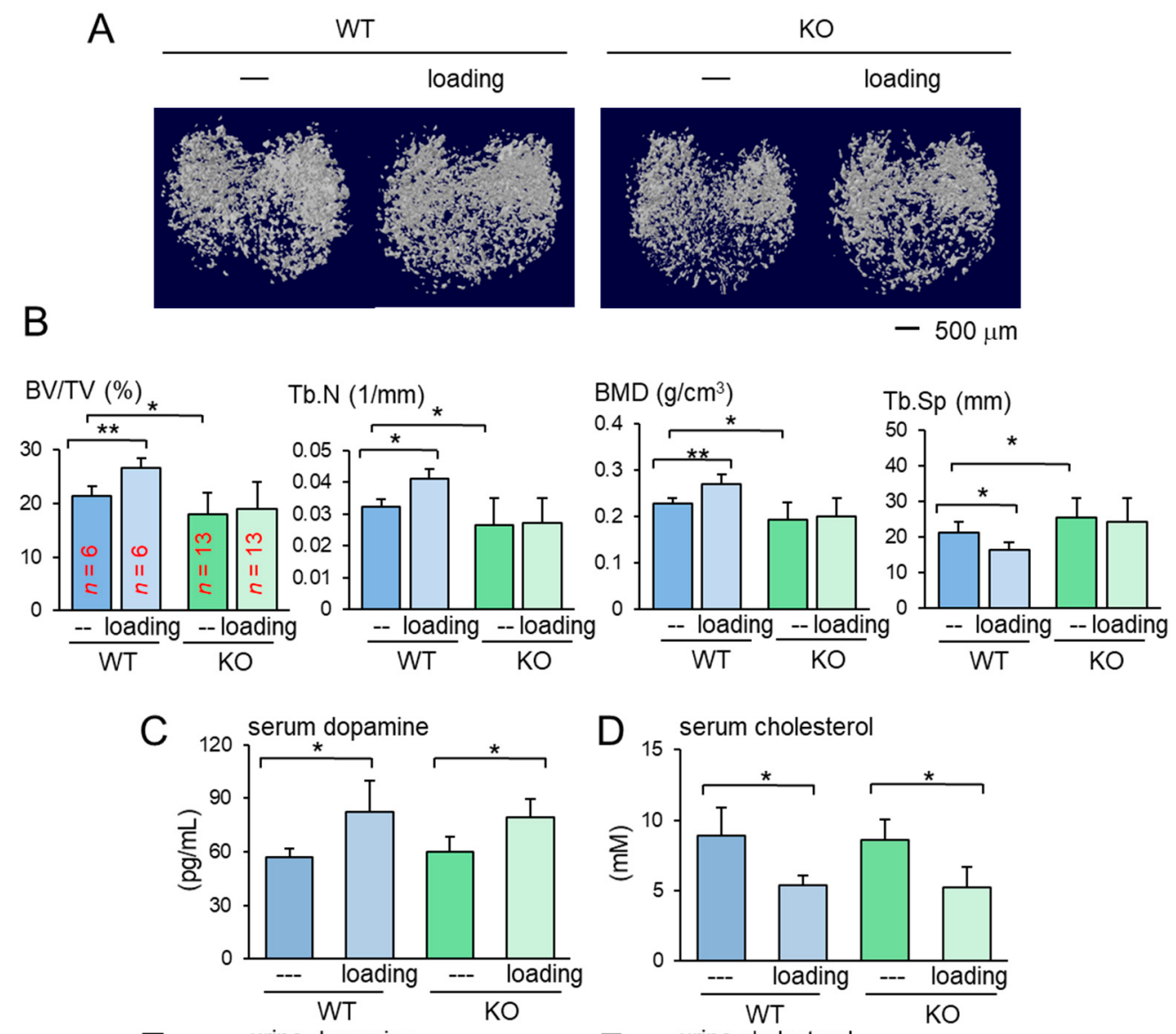

$E$

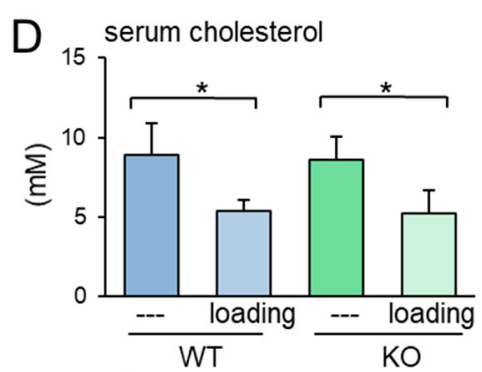

$\mathrm{F}$

urine cholesterol
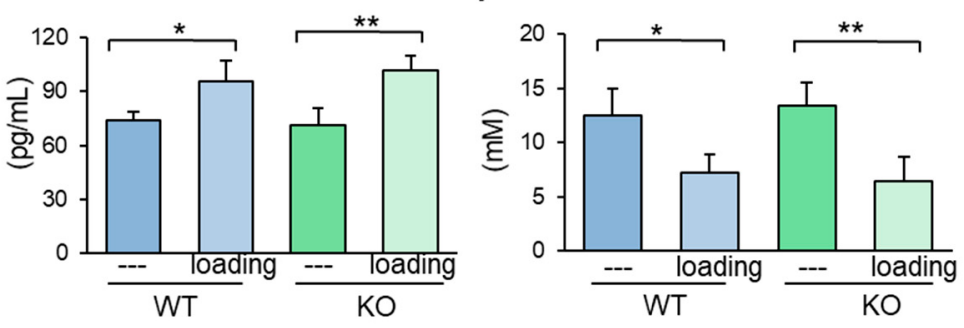

Figure 3. Loading effects on the proximal tibia for the wild-type and knockout mice. $\mathrm{KO}=$ knockout, and WT = wild-type. The data are expressed as the mean \pm S.D. The single and double asterisks indicate $p<0.05$ and 0.01 , respectively. (A) Micro CT images of the proximal tibiae of the wild-type and knockout mice with and without knee loading. No tumor inoculation was applied. (B) Four micro CT-linked parameters in the proximal tibiae in the wild-type and knockout mice without tumor inoculation. BV/TV = bone volume ratio, $\mathrm{Tb} . \mathrm{N}=$ trabecular number, BMD = bone mineral density, and Tb.Sp = trabecular separation. (C,D) Levels of dopamine and cholesterol in the serum in response to tumor inoculation. $(\mathbf{E}, \mathbf{F})$ Levels of dopamine and cholesterol in the urine in response to tumor inoculation.

\subsection{Tumor-Suppressing Effect of TRAIL, p53, Chemerin, and Tumor-Promoting Effect of Nexin}

We next examined the expression of the selected genes including Lrp5 (Wnt coreceptor), MMP2, MMP3, MMP9 (matrix metalloproteinases), Snail (inducer of epithelialmesenchymal transition), Runx2, and TGF $\beta$ (bone-linked tumor promoters), as well as cleaved caspase 3 (apoptosis marker), in response to the selected regulatory proteins in EO771 mammary tumor cells. The administration of recombinant proteins of TRAIL, an apoptosis inducer, and the overexpression of p53, a tumor suppressor, reduced the tumorigenic genes and elevated cleaved caspase 3, an apoptosis marker (Figure 5A,B). The same response was observed to the administration of chemerin recombinant proteins, 
while the opposite response by RNA interference with chemerin siRNA (Figure 5C,D). Consistently, the responses to nexin recombinant protein and nexin siRNA were contrary to those to chemerin counterparts (Figure 5E,F). Regarding TRAMP prostate tumor cells, the trend in their responses to dopamine, cholesterol, TRAIL, p53 plasmids, chemerin, chemerin siRNA, nexin, and nexin siRNA was identical to that of EO771 cells (Figure S5).

A

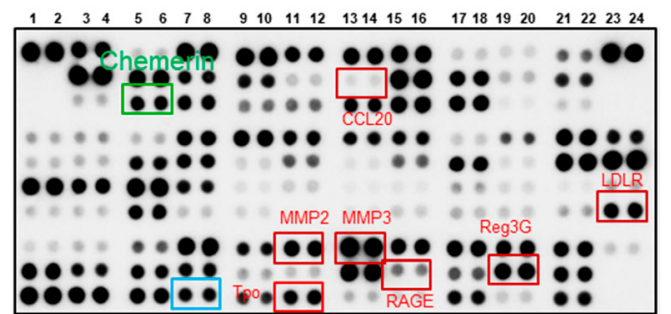

B

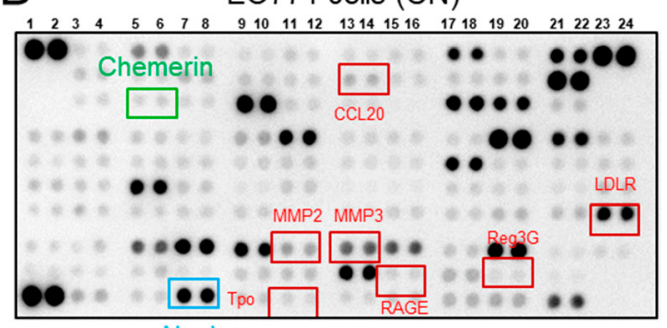

C

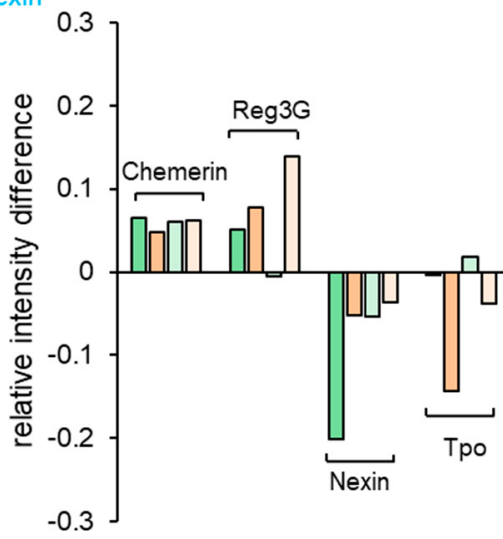

C57BL/6 serum (KO + loading)

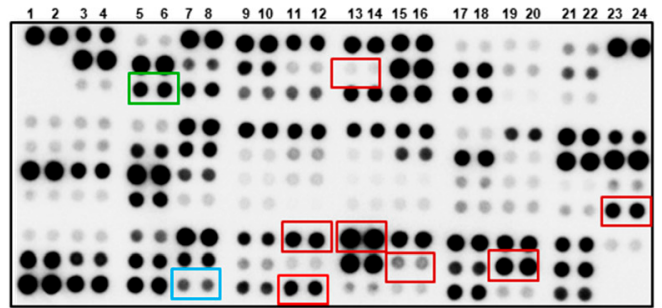

EO771 cells (dopamine)

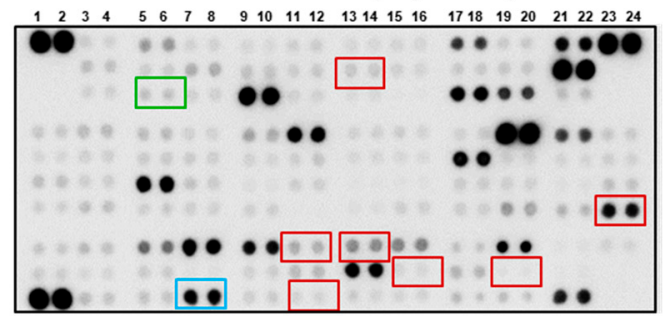

$\square$ KO serum (loading - CN)

$\square$ WT serum (loading - CN)

$\square$ EO771 (dopamine - CN)

$\square$ EO771 (CN - cholesterol)

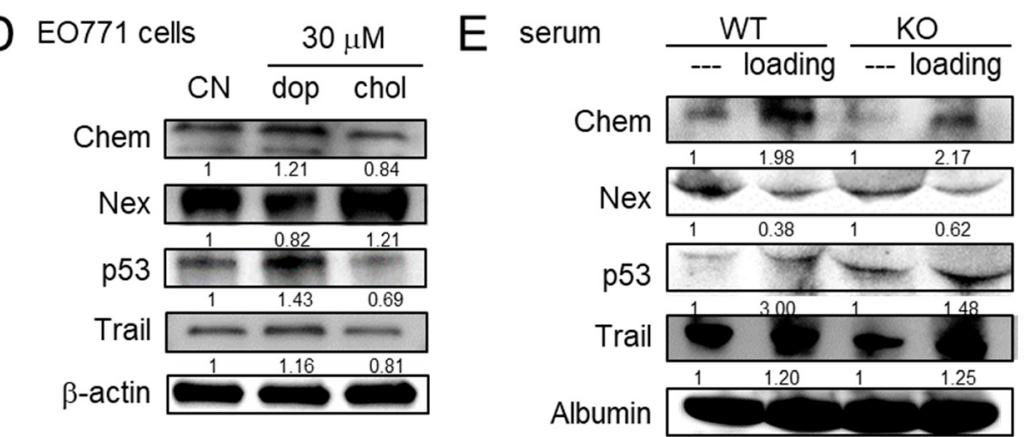

Figure 4. Cytokine array analysis using the serum and EO771 mammary tumor cells. WT = wild-type, KO = knockout, $\mathrm{CN}=$ control, dop = dopamine, chol = cholesterol, Chem = chemerin, and Nex = nexin. (A) Cytokine expression profile in the serum of knockout mice with and without knee loading. (B) Cytokine expression profile in EO771 cells with and without the administration of $10 \mu \mathrm{M}$ dopamine. (C) Summary of the cytokine expression profiles for four pairs of samples, including the sera from the wild-type and knockout mice, and EO771 cells treated with dopamine and cholesterol. (D) Expression of chemerin, nexin, p53, and TRAIL in EO771 cells in response to $30 \mu \mathrm{M}$ dopamine and cholesterol. (E) Expression of chemerin, nexin, p53, and TRAIL in the serum of the wild-type and knockout mice with and without knee loading. 
A EO771

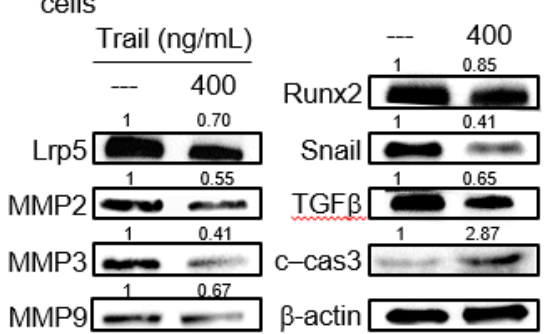

C EO771

cells

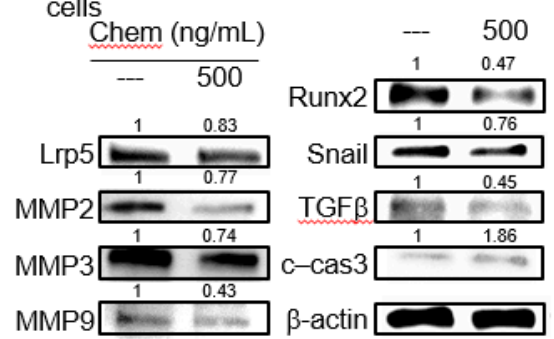

E EO771

cells

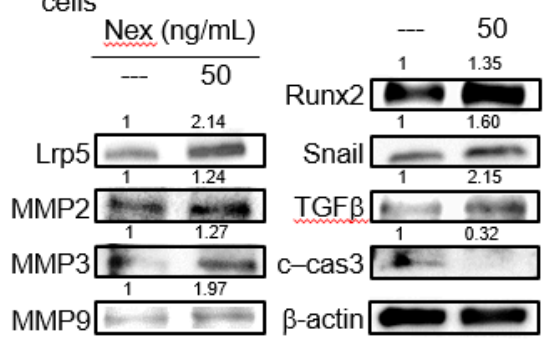

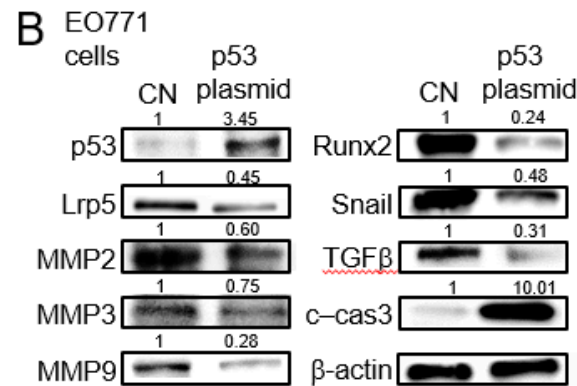

D EO771

cells chem chem

CN SiRNA
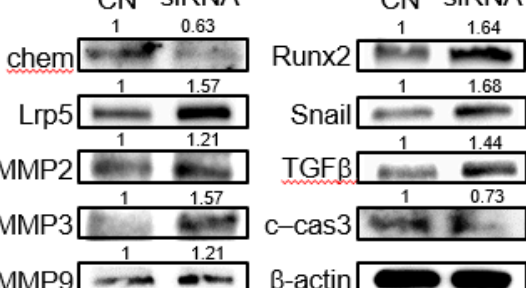

MMP9 $\begin{array}{ccc}1 & 1.21 \\ & \sim & -\infty\end{array}$-actin $\longrightarrow$

F EO771

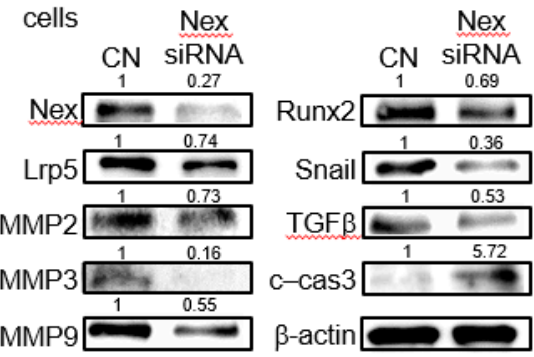

Figure 5. Expression of Lrp5, MMP2, MMP3, MMP9, Runx2, Snail, TGF $\beta$, and cleaved caspase 3 in EO771 mammary tumor cells. $\mathrm{CN}=$ control, c-cas $3=$ cleaved caspase 3 , Chem = chemerin, and Nex = nexin. (A) Response to the administration of TNF-related apoptosis-inducing ligand (TRAIL). (B)Response to the transfection of p53. (C) Response to the administration of chemerin. (D) Response to RNA interference with chemerin siRNA. (E) Response to the administration of nexin. (F) Response to RNA interference with nexin siRNA.

\subsection{Lrp5 as a Tumor-Suppressor in Osteocytes}

To further evaluate the role of Lrp5 in tumor-osteocyte interactions in vitro, we employed Lrp5 plasmids and siRNA. In a spheroid competition assay, the co-culturing of EO771 tumor spheroids with osteocyte spheroids induced the shrinkage of tumor spheroids. Overexpression of Lrp5 in osteocytes enhanced the shrinkage of tumor spheroids (Figure 6A-D). Lrp5-overexpressing osteocyte-derived conditioned medium (CM) also inhibited EdU-based proliferation and scratch-based migration of MDA-MB-231 breast cancer cells and EO771 mammary tumor cells (Figure S6). Furthermore, Lrp5-overexpressing osteocyte-derived CM downregulated Lrp5, MMP9, Runx2, TGF $\beta$, Snail, and nexin with an increase in chemerin in these cells (Figure 6E,F). Consistently, RNA interference with Lrp5 siRNA reversed the response of these genes (Figure 6G-I).

\subsection{Regulation of Osteoclastogenesis by Chemerin and Nexin}

In tumor-driven osteolysis, osteoclasts are responsible for bone resorption. We thus examined the effect of chemerin and nexin on the development of osteoclasts. In response to chemerin, the levels of NFATc1, a master transcription factor of osteoclastogenesis, and cathepsin K, a proteinase involved in bone resorption, were reduced in receptor activator 
of nuclear factor kappa-beta ligand (RANKL)-stimulated RAW264.7 pre-osteoclasts, while the response was opposite to the treatment with nexin (Figure 7A,B). Furthermore, the number of tartrate-resistant acid phosphate (TRAP)-positive multi-nucleated osteoclasts was reduced by chemerin and elevated by nexin (Figure 7C). Collectively, chemerin acted as a suppressor of tumor progression and osteoclastogenesis, whereas nexin as a promoter of tumor growth and osteoclast maturation.

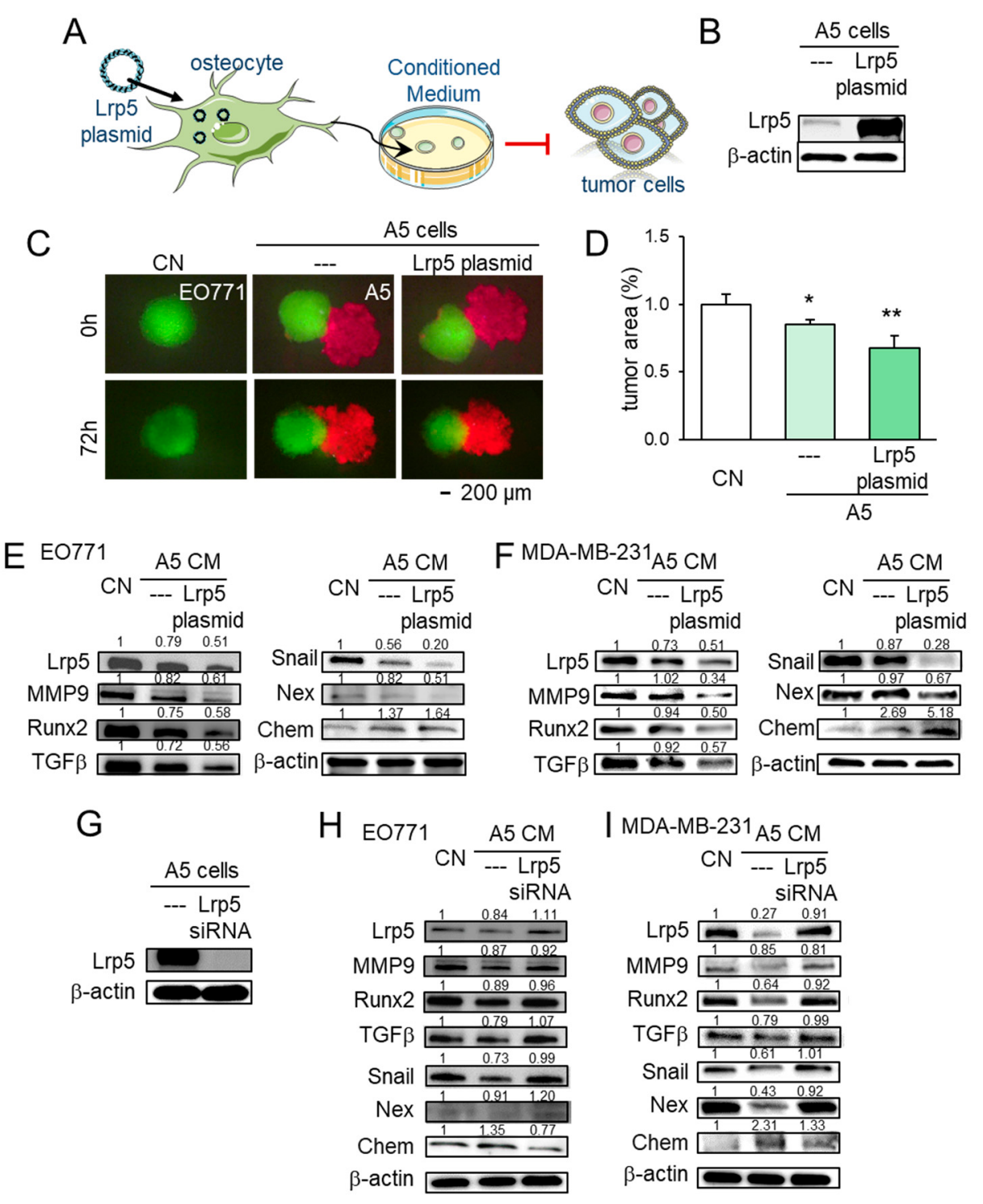

Figure 6. Effect of Lrp5 in osteocytes on EO771 and MDA-MB-231 breast cancer cells. The single and double asterisks indicate $p<0.05$ and 0.01 , respectively. $\mathrm{CN}=$ control, $\mathrm{CM}=$ conditioned medium, Chem = chemerin, Nex = nexin, cat $\mathrm{K}=$ cathepsin $\mathrm{K}$. (A,B) Overexpression of Lrp5 in A5 osteocytes. (C,D) Shrinkage of EO771 tumor spheroids by the incubation with Lrp5-overexpressing osteocytederived CM and osteocytes. (E,F) Altered expression levels of Lrp5, MMP9, Runx2, TGF 3 , Snail, nexin, and chemerin in EO771 and MDA-MB-231 cells in response to A5 osteocyte-derived CM with and without Lrp5 overexpression. (G-I) Altered expression levels of Lrp5, MMP9, Runx2, TGF $\beta$, Snail, nexin, and chemerin in EO771 and MDA-MB-231 cells in response to A5 osteocyte-derived CM in the presence and absence of RNA silencing with Lrp5 siRNA. 
C

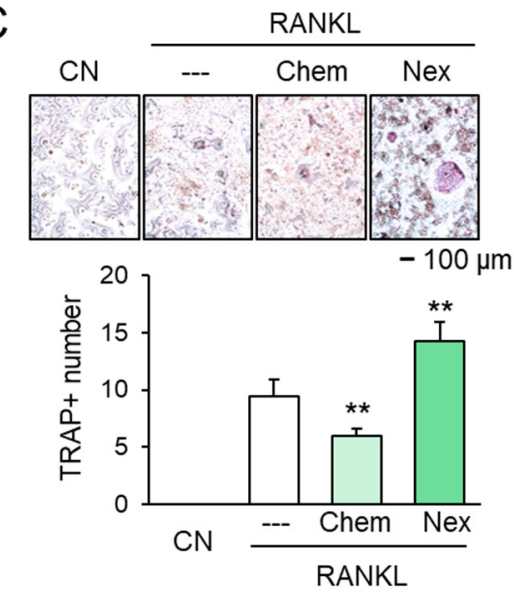

A

A RAW264.7 cells
D $\frac{\text { RANKL }}{-- \text { Chem }}$

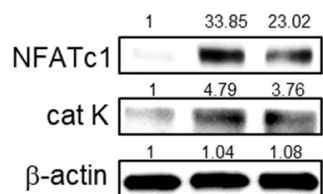

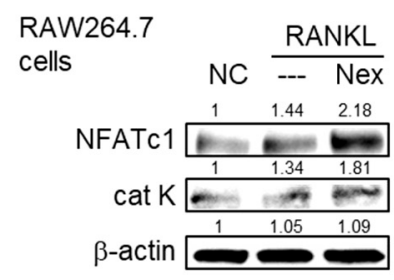
B RAW264.7
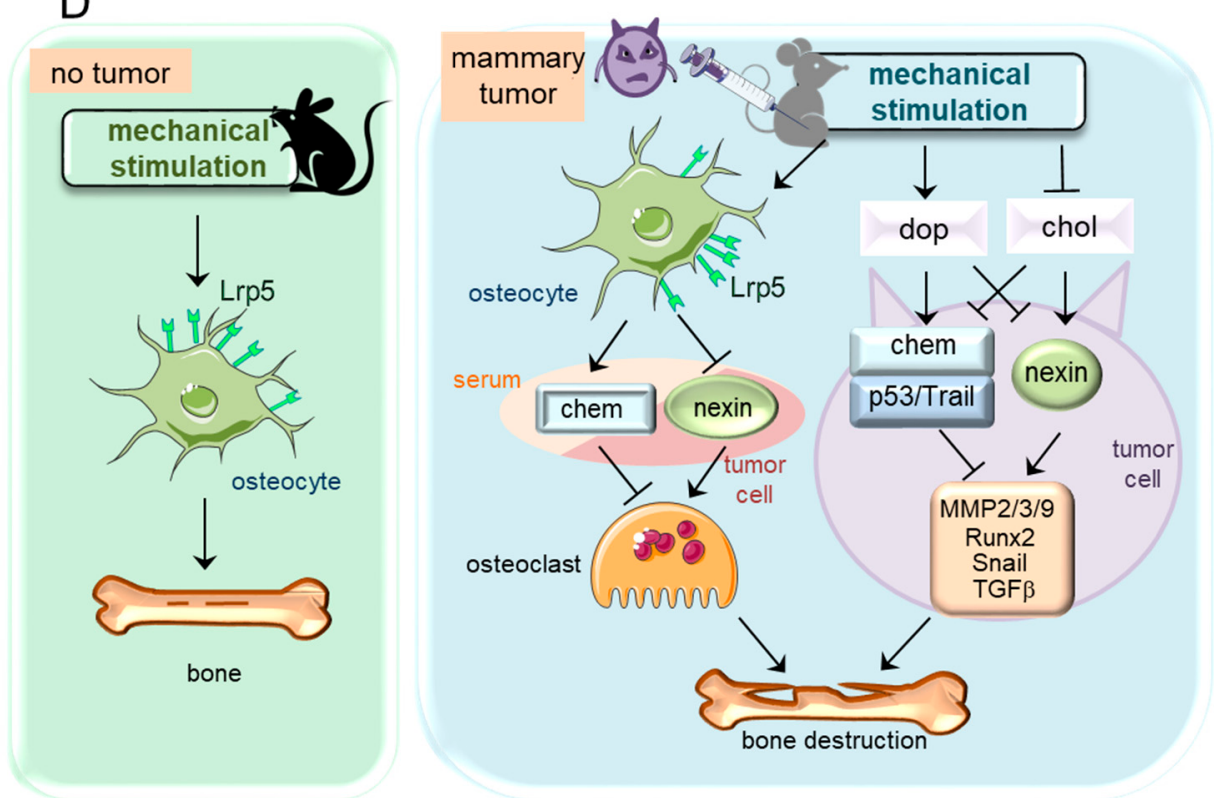

Figure 7. Effects of chemerin and nexin on osteoclasts, and the proposed regulatory mechanism. The double asterisk indicates $p<0.01$. (A,B) Downregulation of NFATc1 and cathepsin K by chemerin and their upregulation by nexin in RANKL-stimulated RAW264.7 osteoclasts. (C) Reduction and elevation of tartrate-resistant acid phosphate (TRAP)-positive cells by chemerin and nexin, respectively. (D) Proposed regulatory mechanism for female mice in response to knee loading. Without tumor inoculation, Lrp5 in osteocytes is necessary to induce loading-driven bone formation. In the tumorinvaded tibia, mechanical loading suppresses bone destruction regardless of Lrp5 in osteocytes. The beneficial loading effects are mediated by the elevation of dopamine and the reduction in cholesterol. Chemerin and nexin act as a suppressor and promotor, respectively, to regulate oncogenic genes such as MMP2, MMP3, MMP9, Runx2, Snail, and TGF 3 .

\section{Discussion}

This study presented that loading-driven tumor suppression was mediated by both Lrp5-independent and Lrp5-dependent mechanisms. The Lrp5-independent mechanism was supported by the observation that regardless of Lrp5 presence in osteocytes, knee loading inhibited tumor growth in the mammary fat pad and bone loss in the tumorinvaded tibia. The Lrp5-dependent mechanism was evident since Lrp5-overexpressing osteocytes inhibited the growth of tumor spheroids and Lrp5-silenced osteocytes upregulated tumorigenic genes such as Runx2, MMP9, Snail, and TGF $\beta$. Because these two mechanisms are present in the wild-type mice, the degree of tumor suppression in the tumor-invaded tibia of the wild-type mice was more significant than that of the knockout 
mice. In support of the Lrp5-independent mechanism, knee loading elevated dopamine and reduced cholesterol in the serum and urine of wild-type and knockout mice. Dopamine was reported to inhibit the oncogenic behavior of tumor cells, while cholesterol was reported to enhance it [23]. The signaling analysis showed that dopamine elevated the levels of tumor suppressors such as chemerin, p53, and TRAIL and reduced the level of nexin, a tumor promoter. By contrast, chemerin was downregulated and nexin was upregulated by cholesterol. Collectively, the present study demonstrated that the Lrp5-dependent and independent loading-driven anti-tumor pathways existed, and both pathways were linked to the upregulation of tumor-suppressing chemerin and the downregulation of tumor-promoting nexin (Figure 7D).

The dual pathway mechanism for loading-driven tumor suppression is different from the mechanism for loading-driven bone formation. Osteocytes are considered mechanosensors in the bone, and it is reported that loading-driven bone formation is promoted by Lrp5-mediated Wnt signaling [24,25]. In this study, we also showed that the loading-driven increase in the bone volume ratio and bone mineral density was observed only in wild-type mice. Notably, however, the loading-driven suppression of tumor growth and tumorinduced osteolysis was observed in both wild-type and knockout mice. The result indicates that Lrp5 in osteocytes is necessary for loading-driven bone formation. For loading-driven tumor suppression, its contribution is significant through tumor-osteocyte interactions, but its absence does not eliminate the loading benefits because of the loading-driven systemic regulation of dopamine and cholesterol. Of note, the status of hormone receptors, such as estrogen receptors, can be involved in the responses to mechanical loading [26,27]. While Lrp5 in tumor cells acts as a tumor promoter in Wnt / $\beta$-catenin signaling that is considered a therapeutic target [28,29], the result in this study indicates that Lrp5 in osteocytes serves as a tumor suppressor. The overexpression of Lrp5 in osteocytes granted the tumor-suppressing capability to osteocytes. The co-culturing of tumor spheroids with Lrp5-overexpressing osteocyte spheroids shrank tumor spheroids, and their $\mathrm{CM}$ downregulated MMP9, Runx2, TGF $\beta$, and Snail. It also inhibited the expression of nexin in tumor cells, while it elevated chemerin, a tumor-suppressor. Conversely, RNA interference with Lrp5 siRNA in osteocytes reversed the responses in tumor cells with a decrease in chemerin and an increase in nexin. Taken together, the role of Lrp5 in Wnt signaling differs in tumor cells and osteocytes, and thus the therapeutic inhibition of Lrp5/Wnt signaling may weaken the intrinsic tumor-suppressing capability of osteocytes.

The elevation of dopamine by physical activities is reported in mouse and human studies [30], but the mechanism of the loading-driven regulation of dopamine, as well as cholesterol, is not well understood in the Lrp5-independent pathway. Dopamine is a neurotransmitter [31], which is synthesized by dopamine neurons in the ventral tegmental area with a rate-limiting enzyme, tyrosine hydroxylase. While dopamine in this study was induced by mechanical loading, it is reported that electrostimulation can also elevate the level of dopamine [32]. The result of this study is consistent with the previous work in which the levels of dopamine and cholesterol were altered in human urine samples after step aerobics [23]. Of note, the physiological levels of dopamine and cholesterol in human serum are reported to be $\sim 40 \mathrm{pg} / \mathrm{mL}$ and $\sim 5 \mathrm{mM}$, respectively [33-35]. The levels we observed for mice in this study were on the same order but slightly higher than the human levels. In the Lrp5-independent mechanism, it is possible that besides dopamine and cholesterol, other neurotransmitters and metabolites are also involved as systemic tumor-regulating agents in the serum and urine.

The protein array analysis for cytokines and chemokines revealed that the levels of chemerin and nexin were mainly altered by knee loading in the serum of wild-type and knockout mice, as well as the responses to dopamine and cholesterol in tumor cells. It is reported that chemerin suppresses hepatocellular carcinoma metastasis, breast cancer growth, prostate cancer progression, skin carcinogenesis, and melanoma [36-40], while nexin is a stimulator of peritoneal metastasis in ovarian cancer [41], as well as the growth, migration, and invasion of breast cancer cells [42]. It is also reported that Wnt signaling 
in tumor cells is inhibited by chemerin while nexin is a downstream target of Wnt signaling [43-45]. We also observed that the levels of p53 and TRAIL were elevated by dopamine and reduced by cholesterol, in which p53 is a well-known tumor suppressor [46], and TRAIL is an apoptosis inducer of tumor cells [47]. Taken together, this study indicates that the upregulation of chemerin, TRAIL, and p53 as well as the downregulation of nexin by mechanical loading contributes to Lrp5-dependent and independent mechanisms by downregulating Lrp5, MMP2, MMP3, MMP9, Runx2, Snail, and TGF $\beta$ in tumor cells.

While this study employed two cancer models using breast and prostate cancer cells, the result may depend on individual types of cancer cells. It is also recommended to evaluate the efficacy of osteocyte-derived CM not only on breast and prostate cancers but also on other cancers. We also observed that the effects of chemerin and nexin on the development of bone-resorbing osteoclasts are consistent with their role in tumor progression. Chemerin suppressed osteoclastogenesis by inhibiting NFATc1 and cathepsin $\mathrm{K}$, while nexin promoted the expression of these two genes. Collectively, the present study showed that chemerin suppresses both tumor progression and RANKL-induced osteoclastogenesis [48], while nexin exerted the opposite responses [49].

\section{Materials and Methods}

\subsection{Cell Culture}

EO771 murine mammary tumor cells (CH3 BioSystems, Amherst, NY, USA, RRID:CVC L_GR23) [50], TRAMP-C2ras murine prostate tumor cells (ATCC, Manassas, VA 20110, USA, RRID:CVCL_3615) [51], and MDA-MB-231 human breast cancer cells (ATCC, RRID:CV CL_0062) [52] were grown in DMEM. MLO-A5 murine osteocytes (obtained from Dr. L. Bonewald at Indiana University, Indiananpolis IN, USA, RRID:CVCL_0P24) and RAW264.7 murine pre-osteoclast cells (ATCC, RRID:CVCL_0493) were grown in $\alpha$ MEM. The culture media were supplemented with $10 \%$ fetal bovine serum and $1 \%$ antibiotics $\left(1 \times 10^{2}\right.$ Units $/ \mathrm{mL}$ penicillin, and $1 \times 10^{2} \mathrm{ug} / \mathrm{mL}$ streptomycin; Life Technologies Corporation, Carlsbad, CA, USA), and cells were maintained at $37^{\circ} \mathrm{C}$ and $5 \% \mathrm{CO}_{2}$. Tumor cells were treated with dopamine (Tocris, Minneapolis, MN, USA), cholesterol (Sigma, Saint Louis, MO, USA), Chemerin recombinant protein,Nexin recombinant protein (R\&D Systems, Minneapolis, MN, USA), and TRAIL recombinant protein (Biolegend, San Diego, CA, USA).

\subsection{MTT, EdU, Invasion, Wound-Healing Scratch, and 3D Spheroid Competition Assay}

Cell viability was examined using an MTT assay (Invitrogen, Carlsbad, CA, USA), and cell proliferation was evaluated by a 5-ethynyl-2'-deoxyuridine (EdU) assay using a fluorescence-based cell proliferation kit (Thermo-Fisher, Waltham, MA, USA) [53]. A Transwell invasion assay and a wound-healing scratch assay were conducted as described previously [54]. A three-dimensional spheroid competition assay was conducted by generating spheroids from EO771 and MLO-A5 cells in ultra-low attachment 96-well plates at concentrations of $1 \times 10^{4}$ cells/well. After $24 \mathrm{~h}$, the EO771 tumor spheroid was transferred to the well of the MLO-A5 osteocyte spheroid. Spheroids were fluorescently stained (Click-iT $^{\mathrm{TM}}$ EdU Alexa Fluor ${ }^{\mathrm{TM}} 488$ Imaging Kit; Thermo-Fisher, Waltham, MA 02451, USA).

\subsection{Western Blotting, RNA Interference, Plasmid Transfection}

Western blot analysis was conducted using the procedure previously described [55]. We used antibodies against Lrp5 (5731s, RRID:AB_10705602), MMP2 (87809s, RRID:AB_2800 107), Runx2 (8486s, RRID:AB_10949892), Snail (3879s, RRID:AB_2255011), TGFß (3711s, RRID:AB_2063354), cleaved caspase 3 (9661s, RRID:AB_2341188) (Cell Signaling, Danvers, MA, USA), MMP3 (sc-21732, RRID:AB_627958), MMP9 (sc-393859), cathepsin K (sc-48353, RRID:AB_2087687), NFATc1 (sc-7294, RRID:AB_2152503) (Santa Cruz, Dallas, TX, USA), Nexin (ab222754), Chemerin (ab103153, RRID:AB_10861013) (Abcam, Cambridge, MA, USA), p53 (UJ290170, Invitrogen, Carlsbad, CA, USA), TRAIL (NB500220, NOVUS, Centennial, CO, USA, RRID:AB_10003305), and $\beta$-actin (A5441, Sigma-Aldrich, Saint Louis, MO, USA, RRID:AB_476744). RNA interference was conducted using siRNA specific to 
chemerin (\#82104), nexin (\#150176), and Lrp5 (s69315) (Thermo-Fisher, Waltham, MA 02451, USA), with a negative siRNA (Silencer Select \#1, Thermo-Fisher) using the procedure previously described [56]. The overexpression of Lrp5 and p53 was achieved by transfecting Lrp5 plasmids (\#115907, Addgene, Cambridge, MA, USA, RRID:Addgene_115907), and p53 plasmids (\#69003, Addgene, Cambridge, MA, USA, RRID:Addgene_69003), respectively.

\subsection{ELISA and Protein Array Analysis}

The levels of cholesterol and dopamine in the mouse urine and serum were determined using ELISA kits (MyBioSource, San Diego, CA, USA). We also employed a mouse XL cytokine array (R\&D Systems, Minneapolis, MN, USA) and determined the expression levels of 111 cytokines and chemokines in the serum of the wild-type and knockout mice, as well as the protein extracts of EO771 mammary tumor cells.

\subsection{Osteoclast Differentiation Assay}

Using RAW264.7 pre-osteoclast cells (ATCC), an osteoclast differentiation assay was conducted in 12-well plates. During a seven-day assay, cells were grown with $20 \mathrm{ng} / \mathrm{mL}$ of RANKL, and the culture medium was exchanged once on day 4. Adherent cells were then fixed and stained with a tartrate-resistant acid phosphate (TRAP)-staining kit (SigmaAldrich, Louis, MO, USA), according to the manufacturer's instructions. TRAP-positive multinucleated cells (above 3 nuclei) were identified as mature osteoclasts and counted.

\subsection{Animal Models}

The experimental procedures were approved by the Indiana University Animal Care and Use Committee (protocol code: SC292R; date of approval: 30 May 2019) and complied with the Guiding Principles in the Care and Use of Animals endorsed by the American Physiological Society. C57BL/six mice lacking Lrp5 in osteocytes (Dmp1-Cre; Lrp5f/f, RRID:MGI: 5014233) were created by breeding Dmp1-Cre transgenic mice with Lrp5 floxed mice, both of which have been described earlier [57]. Mice were housed four per cage and provided with mouse chow and water ad libitum.

In the mouse mammary tumor model, C57BL/six wild-type and knockout female mice ( six weeks, Envigo RMS, Inc., Indianapolis, IN, USA) received subcutaneous injections of EO771 cells $\left(3 \times 10^{5}\right.$ cells in $50 \mu \mathrm{L}$ PBS) to the mammary fat pad on day one. The animals were sacrificed on day 14 , and the weight of each tumor was measured. In the wild-type and knockout mouse model of tibial osteolysis, C57BL/six male mice received an injection of TRAMP cells $\left(3.0 \times 10^{5}\right.$ cells in $20 \mu \mathrm{L}$ PBS $)$ to the left tibia as an intra-tibial injection, and C57BL/six female mice received an injection of EO771 cells $\left(2.5 \times 10^{5}\right.$ cells in $20 \mu \mathrm{L}$ PBS). The animals were sacrificed on day 14 . We harvested the tibiae for histology and $\mu \mathrm{CT}$ imaging, and the blood for ELISA.

\subsection{Knee Loading}

Knee loading was applied daily using an ElectroForce device (TA Instruments, New Castle, DE, USA). Mice were randomly assigned to the placebo and loading groups. Mice in the loading group were anesthetized with $\sim 1.5 \%$ isoflurane, and sinusoidal loads of $1 \mathrm{~N}$ (peak-to-peak) at $2 \mathrm{~Hz}$ were given to the left knee for $5 \mathrm{~min}$. Mice in the placebo group were anesthetized and placed on the loading device without receiving dynamic loads. No adverse effects in response to knee loading were observed.

\section{8. $\mu$ CT Imaging and Histology}

The tibiae were harvested for $\mu \mathrm{CT}$ imaging and histology. Micro-CT was performed using Skyscan 1172 (Bruker-MicroCT, Kontich, Belgium). Using manufacturer-provided software, scans were performed at pixel size $8.99 \mu \mathrm{m}$, and the images were reconstructed (nRecon v1.6.9.18, Bruker-MicroCT) and analyzed (CTan v1.13, Bruker-MicroCT). We focused on the proximal tibia, $1 \mathrm{~mm}$ thick along the length of the tibia, distal to the growth 
plate. In histology, H\&E staining and immunohistochemistry were conducted as described previously $[54,58]$. The samples were blinded for data analysis.

\subsection{Statistical Analysis}

For cell-based experiments, three or four independent experiments were conducted, and data were expressed as mean \pm S.D. In animal experiments, the sample size in the mouse model was chosen to achieve a power of $80 \%$ with $p<0.05$. The primary experimental outcome was tumor weight for the mammary fat pad experiment and the bone volume ratio (BV/TV) for the tibia experiment. Statistical significance was evaluated using a one-way analysis of variance. Post hoc statistical comparisons with control groups were performed using Bonferroni correction with statistical significance at $p<0.05$. The single and double asterisks in the figures indicate $p<0.05$ and $p<0.01$, respectively.

\section{Conclusions}

Our work demonstrates the function of mechanical loading in the suppression of tumor growth; however, the study has a few limitations. We employed three tumor cell lines-two for breast cancer and one for prostate cancer-but the responses to mechanical loading may depend on the types of cancers and the status of hormone receptors of breast cancers. Since the bone size is significantly different between rodents and humans, the loading conditions such as loading forces need to be reevaluated for clinical translations. Further studies are necessary to evaluate the role of the nervous and endocrine systems in the regulation of dopamine and cholesterol in response to mechanical loading. In conclusion, we observed Lrp5-dependent and independent mechanisms for loading-driven tumor suppression. This study revealed that Lrp5 in osteocytes is beneficial to reduce tumor growth in the bone, but loading-driven suppression of tumor growth can take place even in the absence of Lrp5 in osteocytes via the regulation of dopamine and cholesterol. Five-min knee loading sharply altered the levels of cholesterol and dopamine in the mouse serum and urine. The regulation of dopaminergic and cholesterol signaling might provide a novel strategy to restrain tumor growth in breast cancer and bone metastasis associated with breast cancer and prostate cancer. The results indicate the possibility of developing a novel loading-driven and osteocyte-assisted approach for treating bone loss associated with breast and prostate cancers.

Supplementary Materials: The following are available online at https://www.mdpi.com/2072-6 694/13/2/267/s1, Figure S1: Loading-driven reduction in mammary tumors and the protection of tumor-invaded tibia, Figure S2: Effect of knee loading to the tibial cortical bone without tumor inoculation, Figure S3: Cytokine array analysis using the serum and EO771 mammary tumor cells, Figure S4: Responses of EO771 mammary tumor cells to chemerin and nexin, Figure S5: Expression of Lrp5, MMP2, MMP3, MMP9, Runx2, Snail, TGF $\beta$, and cleaved caspase 3 in TRAMP prostate tumor cells, and Figure S6: Effects of Lrp5-overexpressing osteocyte-derived conditioned medium on the proliferation and migration of tumor cells.

Author Contributions: Conceptualization and experiment design, Y.F., S.L., B.L. and H.Y.; data collection and interpretation, Y.F., S.L., R.Z., X.S. and K.L.; writing and drafting manuscript, Y.F., A.R., B.L. and H.Y. All authors have read and agreed to the published version of the manuscript.

Funding: This research was funded by National Institute of Arthritis and Musculoskeletal and Skin Diseases (R01AR052144), National Cancer Institute (R03CA238555), and National Institute of Arthritis and Musculoskeletal and Skin Diseases (R01AR053237) (AGR).

Data Availability Statement: The data presented in this study are available in this article (and Supplementary Material).

Acknowledgments: The authors appreciate Misato Hase for technical assistance.

Conflicts of Interest: The authors declare no conflict of interest. 


\section{References}

1. Schneider, A.P., 2nd; Zainer, C.M.; Kubat, C.K.; Mullen, N.K.; Windisch, A.K. The breast cancer epidemic: 10 facts. Linacre Q. 2014, 81, 244-277. [CrossRef] [PubMed]

2. Castillejos-Molina, R.A.; Gabilondo-Navarro, F.B. Prostate cancer. Salud Publica Mexico 2016, 58, 279-284. [CrossRef] [PubMed]

3. Haider, M.T.; Smit, D.J.; Taipaleenmaki, H. The endosteal niche in breast cancer bone metastasis. Front. Oncol. 2020, 10, 335. [CrossRef] [PubMed]

4. Fornetti, J.; Welm, A.L.; Stewart, S.A. Understanding the bone in cancer metastasis. J. Bone Miner. Res. 2018, 33, 2099-2113. [CrossRef] [PubMed]

5. Kamiya, N.; Suzuki, H.; Endo, T.; Yano, M.; Naoi, M.; Nishimi, D.; Kawamura, K.; Imamoto, T.; Ichikawa, T. Clinical usefulness of bone markers in prostate cancer with bone metastasis. Int. J. Urol. Off. J. Jpn. Urol. Assoc. 2012, 19, 968-979. [CrossRef]

6. Lynch, M.E.; Brooks, D.; Mohanan, S.; Lee, M.J.; Polamraju, P.; Dent, K.; Bonassar, L.J.; van der Meulen, M.C.; Fischbach, C. In vivo tibial compression decreases osteolysis and tumor formation in a human metastatic breast cancer model. J. Bone Miner. Res. 2013, 28, 2357-2367. [CrossRef]

7. Amin, S. Mechanical factors and bone health: Effects of weightlessness and neurologic injury. Curr. Rheumatol. Rep. 2010, 12, 170-176. [CrossRef]

8. Goodman, C.A.; Hornberger, T.A.; Robling, A.G. Bone and skeletal muscle: Key players in mechanotransduction and potential overlapping mechanisms. Bone 2015, 80, 24-36. [CrossRef]

9. Moore, E.R.; Zhu, Y.X.; Ryu, H.S.; Jacobs, C.R. Periosteal progenitors contribute to load-induced bone formation in adult mice and require primary cilia to sense mechanical stimulation. Stem Cell Res. Ther. 2018, 9, 190. [CrossRef]

10. Liphardt, A.M.; Windahl, S.H.; Sehic, E.; Hannemann, N.; Gustafsson, K.L.; Bozec, A.; Schett, G.; Engdahl, C. Changes in mechanical loading affect arthritis-induced bone loss in mice. Bone 2020, 131, 115149. [CrossRef]

11. Yang, S.; Liu, H.; Zhu, L.; Li, X.; Liu, D.; Song, X.; Yokota, H.; Zhang, P. Ankle loading ameliorates bone loss from breast cancer-associated bone metastasis. Off. Publ. Fed. Am. Soc. Exp. Biol. 2019, 33, 10742-10752. [CrossRef]

12. Wang, X.; He, Y.; Tian, S.; Zhu, F.; Huang, B.; Zhang, J.; Chen, Z.; Wang, H. Fluid shear stress increases osteocyte and inhibits osteoclasts via downregulating receptor-activator of nuclear factor kappaB (RANK)/osteoprotegerin expression in myeloma microenvironment. Med Sci. Monit. Int. Med J. Exp. Clin. Res. 2019, 25, 5961-5968. [CrossRef]

13. Takao, S.; Taya, M.; Chiew, C. Mechanical stress-induced cell death in breast cancer cells. Biol. Open 2019, 8. [CrossRef] [PubMed]

14. Norwitz, N.G.; Mota, A.S.; Misra, M.; Ackerman, K.E. LRP5, Bone density, and mechanical stress: A case report and literature review. Front. Endocrinol. 2019, 10, 184. [CrossRef] [PubMed]

15. Kang, K.S.; Hong, J.M.; Horan, D.J.; Lim, K.E.; Bullock, W.A.; Bruzzaniti, A.; Hann, S.; Warman, M.L.; Robling, A.G. Induction of Lrp5 HBM-causing mutations in Cathepsin-K expressing cells alters bone metabolism. Bone 2019, 120, 166-175. [CrossRef] [PubMed]

16. Sawakami, K.; Robling, A.G.; Ai, M.; Pitner, N.D.; Liu, D.; Warden, S.J.; Li, J.; Maye, P.; Rowe, D.W.; Duncan, R.L.; et al. The Wnt co-receptor LRP5 is essential for skeletal mechanotransduction but not for the anabolic bone response to parathyroid hormone treatment. J. Biol. Chem. 2006, 281, 23698-23711. [CrossRef]

17. Saxon, L.K.; Jackson, B.F.; Sugiyama, T.; Lanyon, L.E.; Price, J.S. Analysis of multiple bone responses to graded strains above functional levels, and to disuse, in mice in vivo show that the human Lrp5 G171V High Bone Mass mutation increases the osteogenic response to loading but that lack of Lrp5 activity reduces it. Bone 2011, 49, 184-193. [CrossRef]

18. Park, S.H.; Eber, M.R.; Shiozawa, Y. Models of prostate cancer bone metastasis. Methods Mol. Biol. 2019, 1914, 295-308. [CrossRef]

19. Li, X.; Liu, D.; Li, J.; Yang, S.; Xu, J.; Yokota, H.; Zhang, P. Wnt3a involved in the mechanical loading on improvement of bone remodeling and angiogenesis in a postmenopausal osteoporosis mouse model. Off. Publ. Fed. Am. Soc. Exp. Biol. 2019, 33, 8913-8924. [CrossRef]

20. Zengin, A.; Zhang, L.; Herzog, H.; Baldock, P.A.; Sainsbury, A. Neuropeptide Y and sex hormone interactions in humoral and neuronal regulation of bone and fat. Trends Endocrinol. Metab. 2010, 21, 411-418. [CrossRef]

21. Igwe, J.C.; Jiang, X.; Paic, F.; Ma, L.; Adams, D.J.; Baldock, P.A.; Pilbeam, C.C.; Kalajzic, I. Neuropeptide Y is expressed by osteocytes and can inhibit osteoblastic activity. J. Cell. Biochem. 2009, 108, 621-630. [CrossRef] [PubMed]

22. Liu, S.; Wu, D.; Sun, X.; Fan, Y.; Zha, R.; Jalali, A.; Teli, M.; Sano, T.; Siegel, A.; Sudo, A.; et al. Mechanical stimulations can inhibit local and remote tumor progression by downregulating WISP1. Off. Publ. Fed. Am. Soc. Exp. Biol. 2020, 34, 12847-12859. [CrossRef] [PubMed]

23. Wu, D.; Fan, Y.; Liu, S.; Woollam, M.D.; Sun, X.; Murao, E.; Zha, R.; Prakash, R.; Park, C.; Siegel, A.P.; et al. Loading-induced antitumor capability of murine and human urine. Off. Publ. Fed. Am. Soc. Exp. Biol. 2020, 34, 7578-7592. [CrossRef] [PubMed]

24. Qin, L.; Liu, W.; Cao, H.; Xiao, G. Molecular mechanosensors in osteocytes. Bone Res. 2020, 8, 23. [CrossRef] [PubMed]

25. Kang, K.S.; Robling, A.G. New insights into Wnt-Lrp5/6-beta-catenin signaling in mechanotransduction. Front. Endocrinol. 2014, 5, 246. [CrossRef]

26. Castillo, A.B.; Triplett, J.W.; Pavalko, F.M.; Turner, C.H. Estrogen receptor-beta regulates mechanical signaling in primary osteoblasts. Am. J. Physiol. Endocrinol. Metab. 2014, 306, E937-E944. [CrossRef]

27. Swift, S.N.; Swift, J.M.; Bloomfield, S.A. Mechanical loading increases detection of estrogen receptor-alpha in osteocytes and osteoblasts despite chronic energy restriction. J. Appl. Physiol. 2014, 117, 1349-1355. [CrossRef] 
28. Lu, W.; Liu, C.C.; Thottassery, J.V.; Bu, G.; Li, Y. Mesd is a universal inhibitor of Wnt coreceptors LRP5 and LRP6 and blocks Wnt/beta-catenin signaling in cancer cells. Biochemistry 2010, 49, 4635-4643. [CrossRef]

29. El-Sahli, S.; Xie, Y.; Wang, L.; Liu, S. Wnt signaling in cancer metabolism and immunity. Cancers 2019, 11, 904. [CrossRef]

30. Shimojo, G.; Joseph, B.; Shah, R.; Consolim-Colombo, F.M.; De Angelis, K.; Ulloa, L. Exercise activates vagal induction of dopamine and attenuates systemic inflammation. Brain Behav. Immun. 2019, 75, 181-191. [CrossRef]

31. Runegaard, A.H.; Fitzpatrick, C.M.; Woldbye, D.P.D.; Andreasen, J.T.; Sorensen, A.T.; Gether, U. Modulating dopamine signaling and behavior with chemogenetics: Concepts, progress, and challenges. Pharmacol. Rev. 2019, 71, 123-156. [CrossRef] [PubMed]

32. Warner, R.L.; Johnston, C.; Hamilton, R.; Skolnick, M.H.; Wilson, O.B. Transcranial electrostimulation effects on rat opioid and neurotransmitter levels. Life Sci. 1994, 54, 481-490. [CrossRef]

33. Liu, M.; Luo, J. Relationship between peripheral blood dopamine level and internet addiction disorder in adolescents: A pilot study. Int. J. Clin. Exp. Med. 2015, 8, 9943-9948. [PubMed]

34. Suzuki, K.; Izumi, M.; Sakamoto, T.; Hayashi, M. Blood pressure and total cholesterol level are critical risks especially for hemorrhagic stroke in Akita, Japan. Cerebrovasc. Dis. 2011, 31, 100-106. [CrossRef] [PubMed]

35. Martin, M.J.; Hulley, S.B.; Browner, W.S.; Kuller, L.H.; Wentworth, D. Serum cholesterol, blood pressure, and mortality: Implications from a cohort of 361,662 men. Lancet 1986, 2, 933-936. [CrossRef]

36. Li, J.J.; Yin, H.K.; Guan, D.X.; Zhao, J.S.; Feng, Y.X.; Deng, Y.Z.; Wang, X.; Li, N.; Wang, X.F.; Cheng, S.Q.; et al. Chemerin suppresses hepatocellular carcinoma metastasis through CMKLR1-PTEN-Akt axis. Br. J. Cancer 2018, 118, 1337-1348. [CrossRef]

37. Pachynski, R.K.; Wang, P.; Salazar, N.; Zheng, Y.; Nease, L.; Rosalez, J.; Leong, W.I.; Virdi, G.; Rennier, K.; Shin, W.J.; et al. Chemerin Suppresses breast cancer growth by recruiting immune effector cells into the tumor microenvironment. Front. Immunol. 2019, 10, 983. [CrossRef]

38. Dubois-Vedrenne, I.; De Henau, O.; Robert, V.; Langa, F.; Javary, J.; Al Delbany, D.; Vosters, O.; Angelats-Canals, E.; Vernimmen, M.; Luangsay, S.; et al. Expression of bioactive chemerin by keratinocytes inhibits late stages of tumor development in a chemical model of skin carcinogenesis. Front. Oncol. 2019, 9, 1253. [CrossRef]

39. Pachynski, R.K.; Zabel, B.A.; Kohrt, H.E.; Tejeda, N.M.; Monnier, J.; Swanson, C.D.; Holzer, A.K.; Gentles, A.J.; Sperinde, G.V.; Edalati, A.; et al. The chemoattractant chemerin suppresses melanoma by recruiting natural killer cell antitumor defenses. J. Exp. Med. 2012, 209, 1427-1435. [CrossRef]

40. Rennier, K.; Shin, W.J.; Krug, E.; Virdi, G.; Pachynski, R.K. Chemerin reactivates PTEN and suppresses PD-L1 in tumor cells via modulation of a novel CMKLR1-mediated signaling cascade. Clin. Cancer Res. Off. J. Am. Assoc. Cancer Res. 2020, 26, 5019-5035. [CrossRef]

41. Peng, Y.; Kajiyama, H.; Yuan, H.; Nakamura, K.; Yoshihara, M.; Yokoi, A.; Fujikake, K.; Yasui, H.; Yoshikawa, N.; Suzuki, S.; et al. PAI-1 secreted from metastatic ovarian cancer cells triggers the tumor-promoting role of the mesothelium in a feedback loop to accelerate peritoneal dissemination. Cancer Lett. 2019, 442, 181-192. [CrossRef] [PubMed]

42. Xu, J.; Zhang, W.; Tang, L.; Chen, W.; Guan, X. Epithelial-mesenchymal transition induced PAI-1 is associated with prognosis of triple-negative breast cancer patients. Gene 2018, 670, 7-14. [CrossRef] [PubMed]

43. He, W.; Tan, R.; Dai, C.; Li, Y.; Wang, D.; Hao, S.; Kahn, M.; Liu, Y. Plasminogen activator inhibitor-1 is a transcriptional target of the canonical pathway of Wnt/beta-catenin signaling. J. Biol. Chem. 2010, 285, 24665-24675. [CrossRef] [PubMed]

44. Kozlova, N.; Jensen, J.K.; Chi, T.F.; Samoylenko, A.; Kietzmann, T. PAI-1 modulates cell migration in a LRP1-dependent manner via beta-catenin and ERK1/2. Thromb. Haemost. 2015, 113, 988-998. [CrossRef] [PubMed]

45. Liu-Chittenden, Y.; Jain, M.; Gaskins, K.; Wang, S.; Merino, M.J.; Kotian, S.; Kumar Gara, S.; Davis, S.; Zhang, L.; Kebebew, E. RARRES2 functions as a tumor suppressor by promoting beta-catenin phosphorylation/degradation and inhibiting p38 phosphorylation in adrenocortical carcinoma. Oncogene 2017, 36, 3541-3552. [CrossRef]

46. Kaiser, A.M.; Attardi, L.D. Deconstructing networks of p53-mediated tumor suppression in vivo. Cell Death Differ. 2018, 25, 93-103. [CrossRef]

47. Pishavar, E.; Attaranzadeh, A.; Alibolandi, M.; Ramezani, M.; Hashemi, M. Modified PAMAM vehicles for effective TRAIL gene delivery to colon adenocarcinoma: in vitro and in vivo evaluation. Artif. Cells Nanomed. Biotechnol. 2018, 46, S503-S513. [CrossRef]

48. Kim, H.; Lee, J.H.; Lee, S.K.; Song, N.Y.; Son, S.H.; Kim, K.R.; Chung, W.Y. Chemerin treatment inhibits the growth and bone invasion of breast cancer cells. Int. J. Mol. Sci. 2020, 21, 2871. [CrossRef]

49. Jin, G.; Aobulikasimu, A.; Piao, J.; Aibibula, Z.; Koga, D.; Sato, S.; Ochi, H.; Tsuji, K.; Nakabayashi, T.; Miyata, T.; et al. A small-molecule PAI-1 inhibitor prevents bone loss by stimulating bone formation in a murine estrogen deficiency-induced osteoporosis model. FEBS Open Biol. 2018, 8, 523-532. [CrossRef]

50. Brinker, A.E.; Vivian, C.J.; Beadnell, T.C.; Koestler, D.C.; Teoh, S.T.; Lunt, S.Y.; Welch, D.R. Mitochondrial haplotype of the host stromal microenvironment alters metastasis in a non-cell autonomous manner. Cancer Res. 2020, 80, 1118-1129. [CrossRef]

51. Foster, B.A.; Gingrich, J.R.; Kwon, E.D.; Madias, C.; Greenberg, N.M. Characterization of prostatic epithelial cell lines derived from transgenic adenocarcinoma of the mouse prostate (TRAMP) model. Cancer Res. 1997, 57, 3325-3330. [PubMed]

52. Johnstone, C.N.; Pattison, A.D.; Harrison, P.F.; Powell, D.R.; Lock, P.; Ernst, M.; Anderson, R.L.; Beilharz, T.H. FGF13 promotes metastasis of triple-negative breast cancer. Int. J. Cancer 2020, 147, 230-243. [CrossRef] [PubMed]

53. Wang, L.; Wang, Y.; Chen, A.; Jalali, A.; Liu, S.; Guo, Y.; Na, S.; Nakshatri, H.; Li, B.Y.; Yokota, H. Effects of a checkpoint kinase inhibitor, AZD7762, on tumor suppression and bone remodeling. Int. J. Oncol. 2018, 53, 1001-1012. [CrossRef] [PubMed] 
54. Fan, Y.; Jalali, A.; Chen, A.; Zhao, X.; Liu, S.; Teli, M.; Guo, Y.; Li, F.; Li, J.; Siegel, A.; et al. Skeletal loading regulates breast cancer-associated osteolysis in a loading intensity-dependent fashion. Bone Res. 2020, 8, 9. [CrossRef]

55. Li, F.; Chen, A.; Reeser, A.; Wang, Y.; Fan, Y.; Liu, S.; Zhao, X.; Prakash, R.; Kota, D.; Li, B.Y.; et al. Vinculin force sensor detects tumor-osteocyte interactions. Sci. Rep. 2019, 9, 5615. [CrossRef]

56. Liu, S.; Fan, Y.; Chen, A.; Jalali, A.; Minami, K.; Ogawa, K.; Nakshatri, H.; Li, B.Y.; Yokota, H. Osteocyte-driven downregulation of snail restrains effects of Drd2 inhibitors on mammary tumor cells. Cancer Res. 2018, 78, 3865-3876. [CrossRef]

57. Zhao, L.; Shim, J.W.; Dodge, T.R.; Robling, A.G.; Yokota, H. Inactivation of Lrp5 in osteocytes reduces young's modulus and responsiveness to the mechanical loading. Bone 2013, 54, 35-43. [CrossRef]

58. Hamamura, K.; Zhang, P.; Zhao, L.; Shim, J.W.; Chen, A.; Dodge, T.R.; Wan, Q.; Shih, H.; Na, S.; Lin, C.C.; et al. Knee loading reduces MMP13 activity in the mouse cartilage. BMC Musculoskelet. Disord. 2013, 14, 312. [CrossRef] 